https://www.journal-imab-bg.org

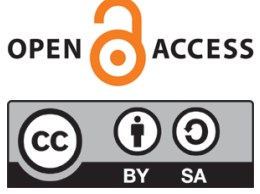

Original article

\title{
ATTITUDES TOWARDS THE USE OF INFORMA- TION AND COMMUNICATION TECHNOLOGIES IN HEALTHCARE
}

\author{
Kristina Kilova $^{1}$, Denis Milkov ${ }^{2}$, Nonka Mateva ${ }^{1}$ \\ 1) Department of Medical Informatics, Biostatistics and e-Learning, Faculty \\ of Public Health, Medical University of Plovdiv, Bulgaria. \\ 2) Clinic of Otorhinolaryngology, University Hospital "Kaspela”, Plovdiv, \\ Bulgaria.
}

\section{ABSTRACT}

The opportunities that information and communication technologies (ICTs) provide in the field of healthcare are many and constantly growing. They enable consumers to access health information and remotely receive consultations and treatment. Also, they help improve health promotion activities whose mission is to establish health as a core value.

The aim of the present study is to examine the attitudes towards the use of ICTs in healthcare.

Materials and Methods: A direct anonymous survey was conducted among 356 respondents, randomly selected and with an average age of $38.26 \pm 0.15$ (110 men and 246 women). The data analysis was performed using statistical software SPSSv.23.0.

Results: The results showed that $91.6 \%$ of respondents have used or are willing to use digital devices to search for health information. A statistical gender difference was found in answer to the question about using a digital device for self-registration of information, as women are more likely to do so. Elderly people are more reserved and hesitant about providing health information to a government agency. Smartphone and computer/laptop are among the most preferred digital modalities in the healthcare field, with men and younger respondents being more likely to use digital devices in relation to their health. Women are more critical about the use of ICTs in healthcare $\left(\chi^{2}=48.2\right.$, $\mathrm{P}<0.05)$.

Conclusion: The present study found a positive attitude among all respondents towards the introduction of ICTs in the healthcare field and the opportunity for a remote contact with medical professionals, using digital devices.

Keywords: healthcare, digital devices, information and communication technologies, health information, telemedicine.

\section{INTRODUCTION}

Global technological developments are gradually entering spheres concerning human health. Today, users have the opportunity to use information and communication technologies (ICTs) to access health information, communicate with healthcare establishments and receive remote consultation and treatment. In this way, modern technologies are able to influence the prevention, treatment and rehabilitation of diseases. The widespread use of mobile smartphones has further increased these trends. According to the World Bank, the mobile subscriptions for the year 2017 in Bulgaria were 8532910 [1]. Therefore, these trends show a possible direction for the allocation of resources towards the development of mobile applications for a number of socially significant diseases.

The opportunities that ICTs provide in the field of healthcare are many and constantly growing. They can help improve health promotion activities, disease prevention and health culture of the population. Facilitated access to health information helps people make informed decisions about their health. Improving the health culture of the population will improve the functioning of the healthcare system as a whole. Health-informed people are much more capable of following recommendations for disease prevention, know when to seek medical attention, and are more compliant to treatment. A higher level of health culture and disease awareness will also facilitate the work of physicians and other healthcare professionals. All these factors could gradually lead to the development of a society with lower incidence and prevalence of the disease, improved treatment outcomes and lower healthcare costs.

For people living outside large cities, the use of ICTs in the healthcare sector is particularly important. Far from medical facilities, they do not seek medical attention for many health problems due to difficult access. This is particularly relevant for elderly people, many of whom have difficulties in travelling for medical care. Modern digital technologies offer unprecedented opportunities for access to health information, remote contact with a medical specialist, and timely medical assistance. For example, telemedicine was successfully applied for medical emergencies in the Himalayas at an altitude of 3,353 meters [2].

ICTs are also potentially applicable in healthcare among socially discriminated and marginalized people. Behind the veil of a digital device, they are able to seek medical help anonymously. In such a way, these people have improved access to medical care.

Digital technologies in healthcare could also improve the quality of medical care by comparing the health status 
of individuals before and after treatment, as well as with other treatment methods. They are able to objectively assess criteria for the health of individuals, groups and the population as a whole. In addition, through the use of ICTs health professionals in developing countries can contact their colleagues in developed countries [3]. Thus, the exchange of experience and knowledge in the prevention and treatment of diseases will be encouraged. Also, the communication between health professionals will improve collaboration in scientific projects and help build the future direction for medical development, according to the needs of the world's population in the current era of globalization.

With the introduction of ICTs in the healthcare sector, the role that healthcare institutions and medical professionals' uptake is of particular importance. It must be based primarily on the attitudes and needs of the served populations. Healthcare institutions and medical professionals must be able to respond to modern trends in the application of new technologies in healthcare. The healthcare system needs to be ready to deploy new ICTs applications in order to improve public health, both on a regulatory and practical level.

Health agencies and healthcare professionals have the responsibility to control the use of ICTs in healthcare. For example, the current state of health information available online is unsatisfactory. Although sometimes of high quality, it is often subjective and incomplete, and sometimes, completely wrong. Such health information damages the health of the individual, and the population leads to unhealthy behaviors and damages the health culture of the population. The medical community must take an active stance to oppose the spread of this type of wrong information by enacting specific measures for its assessment and limitation. Today, more than ever, society needs access to quality health information online.

The prerequisites for the development of ICTs and their capabilities in healthcare are many, but central to their implementation is the society served. The effectiveness of their application depends not only on the size and demographic characteristics of the population but also on the desire to use modern technologies. It is important for the population to be aware of the potential of these new technologies and for their needs to be taken into consideration. Therefore, it is necessary to objectively study peoples' attitudes towards the use of ICTs in healthcare, as well as their needs and willingness to use them for their health.

The aim of the present study is to examine the attitudes towards the use of ICTs in healthcare among the Bulgarian population.

\section{MATERIAL AND METHODS}

Survey method

A direct anonymous survey was conducted among 356 respondents from across the country, who were randomly selected, between October 2018 and April 2019. The average age of respondents was $38.26 \pm 0.15$ years

An author's survey, consisting of two panels, was used. The first panel contained information about the demographic characteristics of gender and age. The second consisted of 14 questions. For eleven of them, a5-step Likert scale was used, for two - an opportunity to answer with more than one answer, and one question asked for the graduation of provided responses.

\section{Statistical methods}

The data analysis was performed using the statistical software SPSS v.23.0. We used the descriptive statistic, t-test for Student and chi-square test for correlation with $\mathrm{P}<0.05$, as statistically significant.

MS Excel 2016 was used for the graphical presentation of the data.

\section{RESULTS}

A total of 356 respondents were surveyed on the use of ICTs in healthcare. Approximately two-thirds of the respondents were women $(n=246 ; 69.1 \%)$.

The respondents were grouped into four age groups: 18-24, 25-49, 50-64 and over 65 years of age, which matched the age categories used by the National Statistical Institute (NSI) of the Republic of Bulgaria (fig.1).

Fig. 1. Distribution of the respondents by age

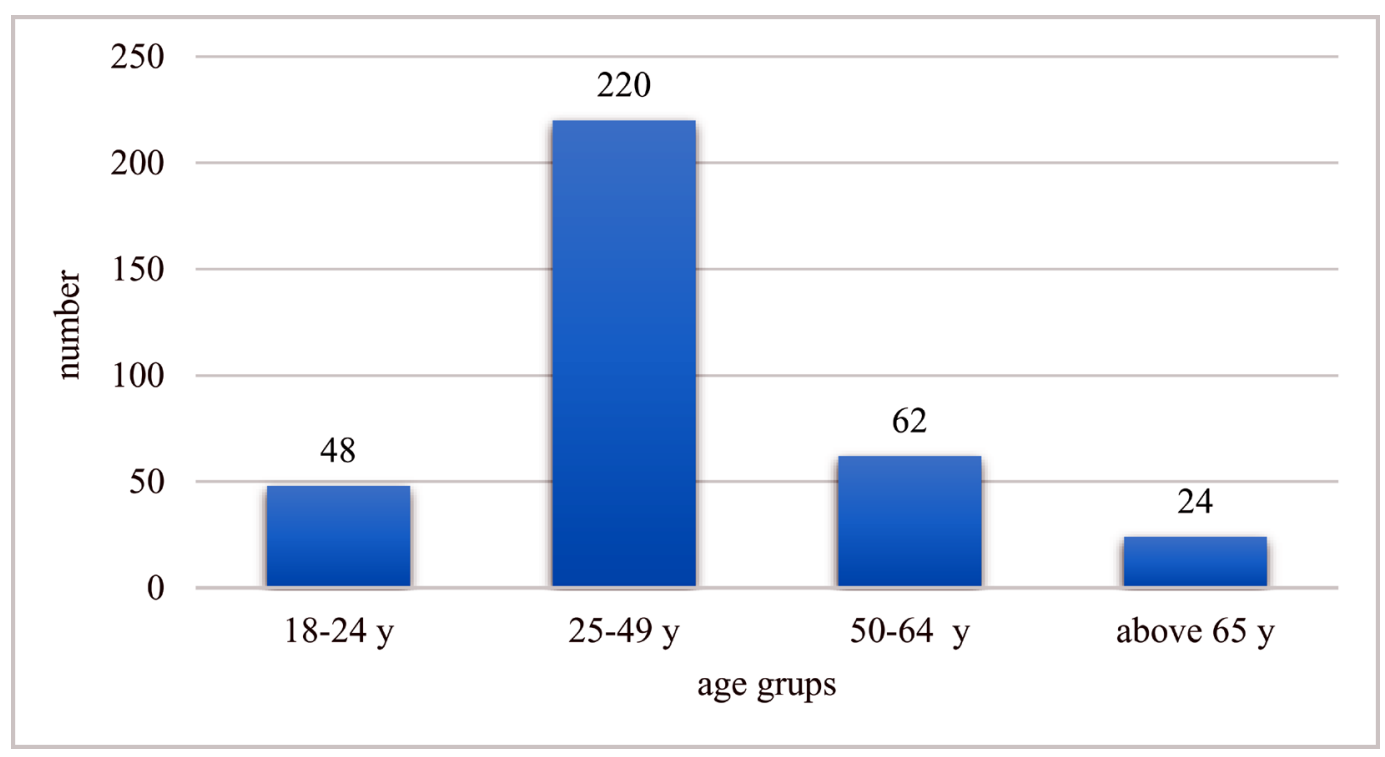


Changes were made for the 15-24 years of the age group which was changed to the 18-24 years of age, as only adult respondents were included in the study, and for the 65-79 years of age and over 80 years of age groups, which were united in the group over 65 years of age.

The results showed that $91.6 \%$ of all respondents answered positively to the previous or future use of a digital device (computer, tablet, smartphone, smartwatch, etc.) to search for information regarding their health. This allows us to conclude that most respondents are already looking for health information through a digital device or are willing to start. An additional study is needed to determine the type of health information that people search for using a digital device. This will allow for the development of appropriate ICTs applications in healthcare that meet the needs of the population.

Most respondents $(79.2 \%)$ have used or would use a digital device for self-registration of information regarding their health. It was found that women $\left(\chi^{2}=11.47 ; \mathrm{P}<0.05\right)$ and younger respondents $\left(\chi^{2}=25.993 ; \mathrm{P}<0.01\right)$ are more likely to register health information. In self-registration of health information, consumers have control over it as they have the freedom to register it at their discretion. Although such information is subjective, it is very useful because health itself can be considered as being subjective, especially regarding present symptoms. To support this type of health information, it is desirable to additionally use health indicators that objectively represent to some extent, the health status.

Nearly $75 \%$ of respondents have used or would use a digital device for registering health information (e.g. pedometer, electrodes, Holter monitor). The results showed that respondents have a positive attitude towards the recording of health information through sensors. Comparison between these results and the results of self-registration of health information shows that older respondents prefer to register health information by sensors rather than manually. This may be due to their confidence in autonomous technologies such as sensors or fears of difficulties with manual registration.

For the most part $(79.2 \%)$, the respondents have shared or are willing to share information about their health from a digital device with a doctor during a visit (fig.2).

Fig. 2. Sharing health information from ICTs with a physician during a consultation visit.

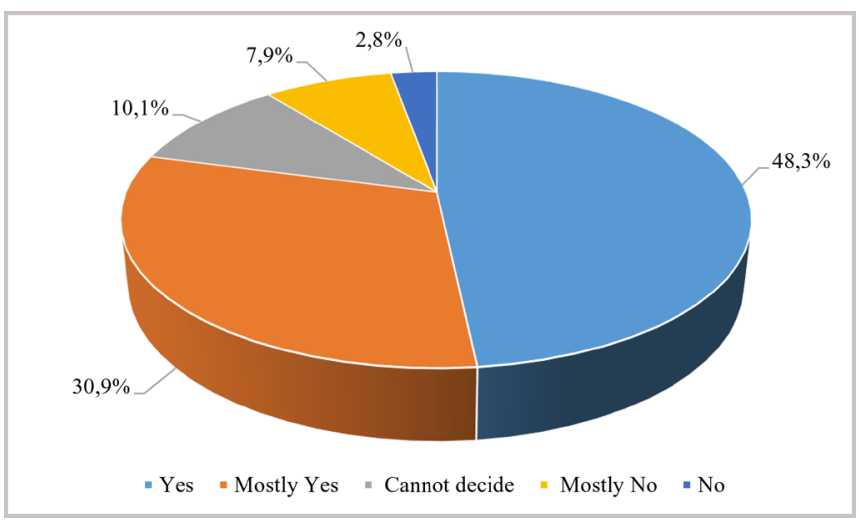

This is a prerequisite for the development of ICTs applications in the healthcare field which collect quality health information to help assess consumer health, prevent and treat illnesses. An important aspect is the need to motivate healthcare professionals in a way that relieves their workload and aids them in the management of patients.

Respondents would also share information about their health from a digital device with a physician in real-time (via an Internet connection). The results follow a distribution closely resembling the responses to sharing health information from ICTs with a doctor during a visit. This shows the positive attitude of respondents towards the availability of immediate contact with a doctor. An important part of the development of this type of doctor-patient communication is dependent on the physician's workload and willingness to participate. ICTs should be used in healthcare in a way that, in addition to exhaustively assessing the condition of the patient, best presents the information to the physician.

The results show a hesitant attitude of respondents regarding the sharing of health information with a government health agency (e.g. Regional Health Inspectorate) using ICTs (fig.3).

Fig. 3. Sharing health information with a government agency through ICTs

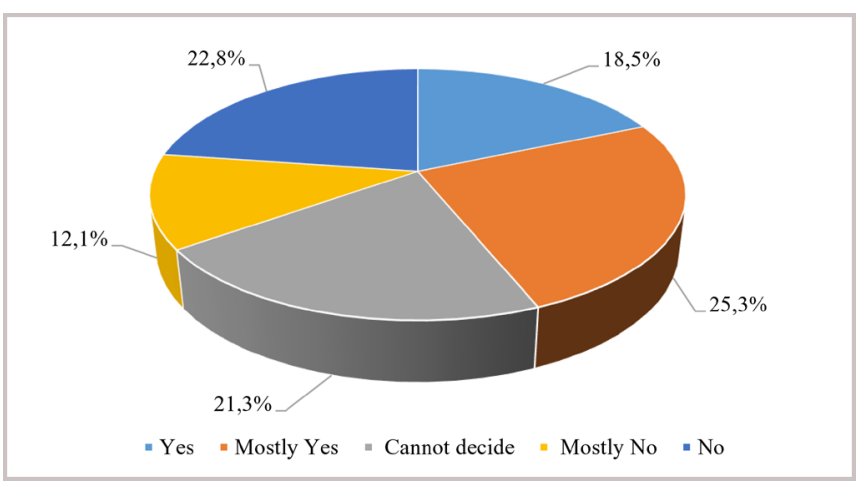

Elderly people are more reserved about providing health information to a government agency $\left(\chi^{2}=29.392\right.$; $\mathrm{P}<0.01)$. This result indirectly reveals the amount of trust that respondents give government agencies regarding their digital health information. To increase confidence, it may be necessary to develop specific programs which clearly explain the ways in which the collected health information will be used, in order to overcome people's inhibitions. Widespread participation is particularly important for the improvement of populational health. Government agencies such as the Regional Health Inspectorates are able to obtain through the use of ICTs quality health information on the basis of which they can develop registers and effective preventive programs for positively influencing the population health.

The opinion of the respondents regarding the sharing of health information with a health insurance agency is split between the two end-points of the scale (National Health Insurance Fund or private insurer). Positive answers give $150(42.1 \%)$ of them, while negative answers - 128 (36\%). More likely to share such information are younger people $\left(\chi^{2}=33.833 ; \mathrm{P}<0.001\right)$. It is noteworthy that more than $20 \%$ of respondents can not decide on an answer to this question. This result may be due to concerns by respondents about how their personal health information will 
be used. As this question was asked generally, it is necessary to further study the propensity of people to share health information with private and government health insurance agencies. In this way, it can be established, for example, that people would share health information with a government health insurance agency, but not with a private, or vice versa.

Over $80 \%$ of respondents would use a digital device for consultation with a physician. This result demonstrates their willingness to facilitate their access to a physician, respectfully to healthcare, through the use of ICTs. An important aspect of teleconsultation is the ability of medical professionals to quickly and remotely respond to patients when needed. Therefore, it is necessary to either develop an appropriate timetable in the workday of physicians allowing teleconsultation or to appoint physicians whose sole task is the teleconsultation of patients.

All this reveals the need to build ICTs systems for the provision of teletherapy. The ICTs systems for teletherapy will give access to healthcare to people away from healthcare establishments. Telemedicine is a field of medicine with a great outlook where opportunities are yet to grow and develop.

The most popular digital modalities for searching, registering and sharing information about respondents' health are smartphones and computers/laptops. These results show that smartphone applications need to be a priority in the development of ICTs in the healthcare sector. Desktop computer and laptop applications should be second in priority. The other digital modalities - smartwatches, specialized portable devices and autonomous sensors - need to be tailored to healthcare as future development of ICT.

More than half of respondents want to use ICT for recording and monitoring arterial blood pressure and pulse, blood glucose, current diagnoses and medications taken, diet and calorie intake, body weight, and physical activity (fig. 4). These results are particularly beneficial as arterial hypertension and diabetes are socially significant diseases.

Fig. 4. Type of health information

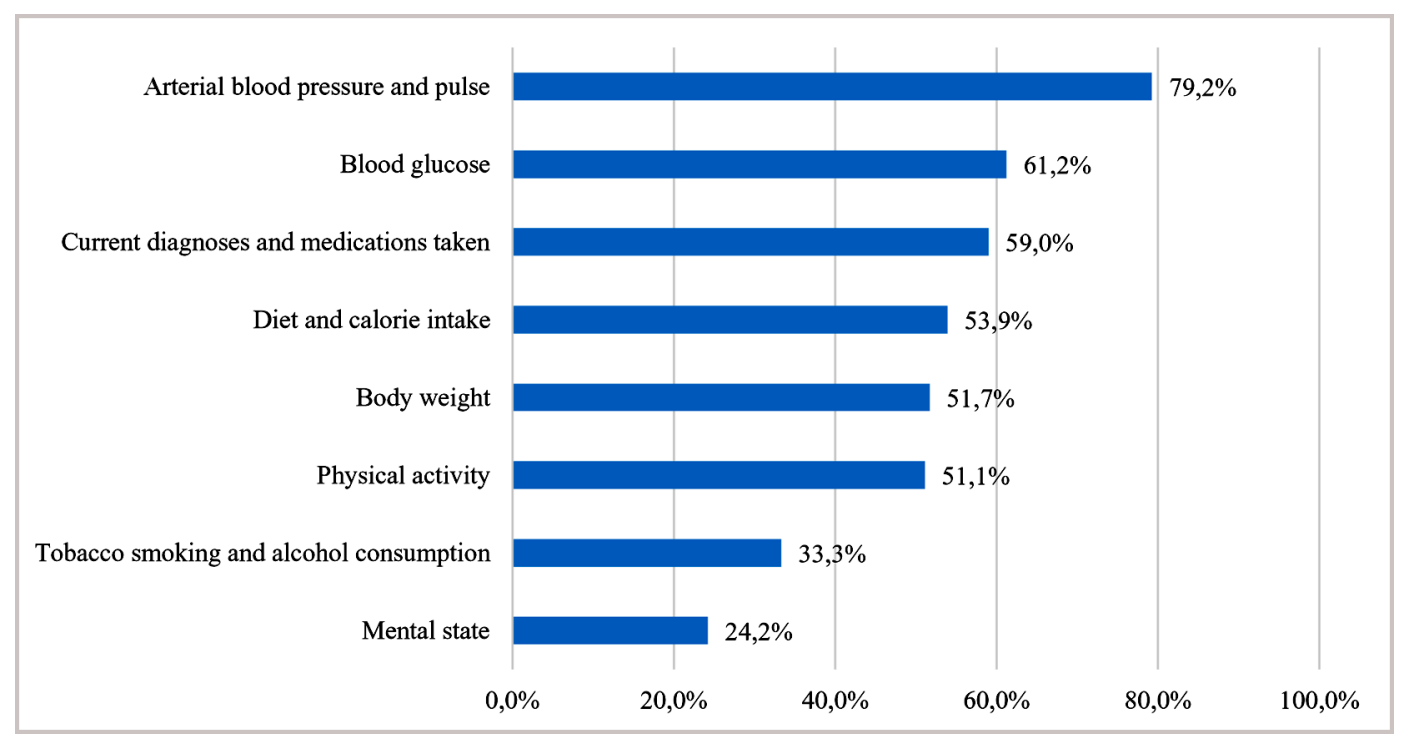

According to our respondents, the most common reason for declining the use of a digital device for searching, registering and sharing personal health information is usage difficulties (57.4\%) (fig. 5).

Fig. 5. Reasons to decline the use of ICTs in healthcare

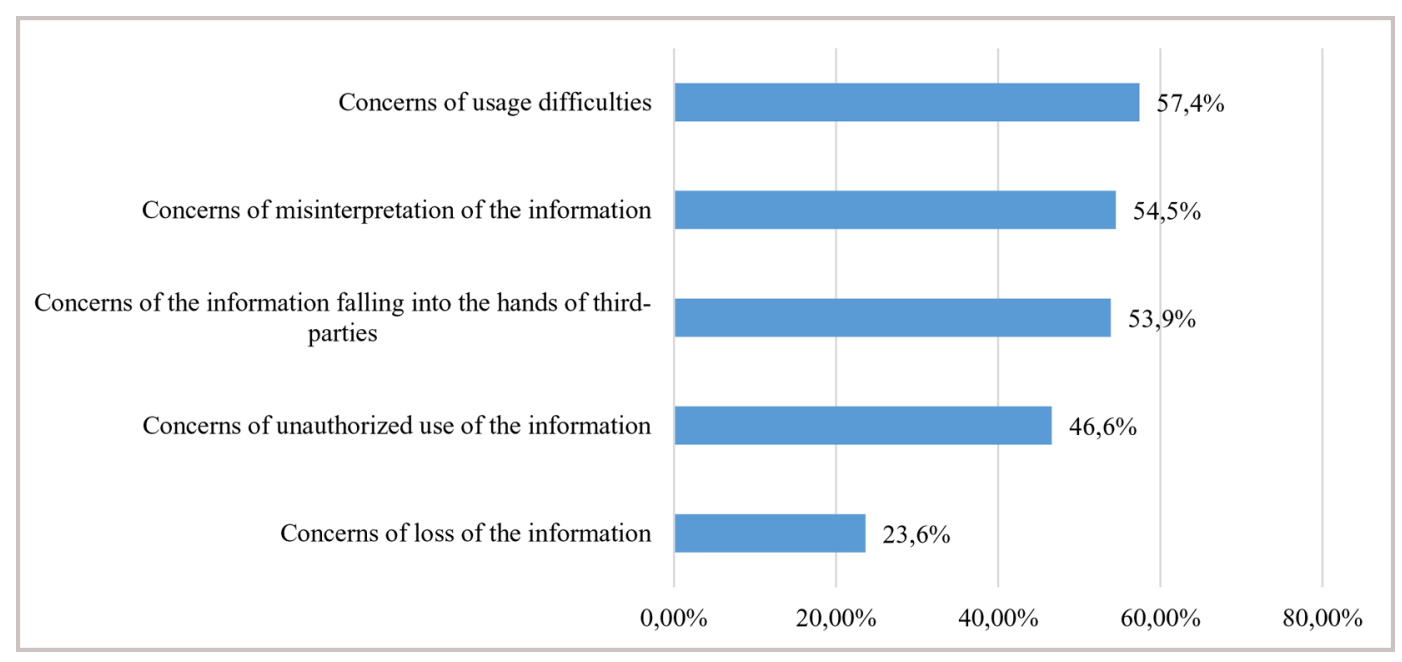


It should be noted that $53.9 \%$ of respondents have concerns about the confidentiality of their personal health information and the possibility of it falling into the hands of third-parties. These concerns are shared by people in other parts of the world.

The overall attitude of the respondents on the application of ICTs in healthcare is very good. Women $\left(\chi^{2}=14.607 ; \mathrm{P}<0.01\right)$ and older respondents $\left(\chi^{2}=39.575\right.$; $\mathrm{P}<0.001)$ are more reserved.

\section{DISCUSSION}

According to a survey conducted by the Pew Research Center, $81 \%$ of US adults by September 2012 use the Internet, and $72 \%$ of them have sought health information online over the past year [4]. This means that, according to the Pew Research Center, $58 \%$ of the surveyed search for health information online which is lower than the result from our study (91.6\%). According to another Pew Research Center survey, only $52 \%$ of mobile smartphone owners have used them to search for health information [5]. This difference may be due to the fact that our survey includes, in addition to people who have used a digital device to search for health information, those who are inclined to start using one.

Our respondents have positive attitudes toward the use of telemedicine services for real-time and remote health monitoring. A study in South Korea among patients, their caregivers and medical professionals found that most would use real-time remote electrocardiographic monitoring [6]. Their respondents' trust in telemedicine is justified because a study on the accuracy of teledermatology and teledermatoscopy in the diagnosis of the most common skin neoplasms in Ukraine found it to be $90.3 \%$ [7].

An important aspect of teleconsultation is the ability of medical professionals to respond to the needs of patients. Therefore, it is necessary to either develop an appropriate timetable in the working day of medical specialists, allowing time for teleconsultation, or to appoint doctors whose sole task is to teleconsult patients.

The results of this study on the attitudes regarding the use of ICTs for arterial blood pressure (79.2\%) are supported by a survey of workers and students in South Korea $(94.3 \%)$. On the other hand, their blood glucose results $(93.6 \%)$ differ from ours $(61.2 \%)$ [8]. Our outcomes regarding attitudes for the use of ICTs for blood glucose support those from the study of Muigg D. et al. on the readiness of patients toward the use of a telemonitoring diabetes system in Austria (65\%) [9]. The use of ICTs for blood glucose is particularly avant-garde. For example, a meta-analysis of 42 randomized controlled trials for HbA1c changes after regular care and telemedicine intervention, found that the decrease in $\mathrm{HbA} 1 \mathrm{c}$ was significantly greater in telemedicine groups, especially when telemedicine programs lasted over 6 months and the patients had type 2 diabetes [10]. The application of ICTs is an opportunity for preven- tion, early diagnosis, registration and more successful treatment of arterial hypertension and diabetes.

The fact that only $24.2 \%$ of respondents would use ICTs for their mental health shows their reluctance to sharing personal socio-psychological states and problems. In Saudi Arabia, an online study among psychiatric patients found that $64 \%$ use their cell phones to track depression and/or anxiety [11]. Global studies on the use of ICTs for mental health show interesting results. For example a study in Poland for the application of smartphones in psychiatry allowing communication with a medical specialist and treatment dosage adjustments from a distance found a significant improvement in the affective, cognitive and schizophrenic symptoms after one year compared to a placebo group [12]. Also, telepsychiatry is increasingly used for patients abusing opioids in the northern parts of Ontario, Canada [13]. This shows that telemedicine is an important tool for providing psychiatric care to people in remote areas.

The respondents in our survey who are willing to share information on tobacco smoking and alcohol use by ICTs are $33.3 \%$. Interestingly, the results of the European Commission for Bulgaria show a $28 \%$ incidence of tobacco smoking.

The results obtained in relation to body weight, nutrition and calorie intake are extremely relevant due to the fact that according to WHO data, the Bulgarian nation has a high incidence of overweight people and obesity. According to the Pew Research Center, physical exercise, diet and bodyweight applications are the most popular smartphone applications for health with $38 \%$ monitoring their physical activity, $31 \%$ diet and $12 \%$ body weight [5]. In general, $27 \%$ of adults in the US use some kind of technology to track a health indicator [14].

For example, a study in South Korea for real-time electrocardiographic monitoring has found that the biggest barrier to the use of these technologies is privacy concerns regarding personal information [6]. Another study, which looked at the readiness of using a telemonitoring system for diabetes among doctors and patients in Austria, found that the biggest concerns are data protection and loss of personal communication [9].

\section{CONCLUSION}

The present study found positive attitudes among all respondents toward the implementation of ICTs in healthcare. The responders want to access digital health information and to be able to remotely contact medical physicians using telemedicine in the forms of teleconsultation and teletherapy.

\section{Acknowledgement}

This research was supported by National Research Program "Electronic Health in Bulgaria" (e-Health) D-01200/16. 11. 2018. 


\section{REFERENCES:}

1. Mobile cellular subscriptions. Data World Bannk. 2018. [Internet].

2. Ganapathy K, Alagappan D, Rajakumar H, Dhanapal B, Subbu GR, Nukala L, et al. Tele-Emergency Services in the Himalayas. Telemed $J E$ Heal. 2019 May;25(5):380-390. [PubMed] [Crossref]

3. McLellan F. Information technology can benefit developing countries. Lancet. 2001 Jul 28; 358(9278):308. [PubMed] [Crossref]

4. Fox S, Duggan M. Health Online 2013. Pew Res Cent. January 15, 2013. [nternet].

5. Fox S, Duggan M. Mobile Health 2012. Pew Res Cent. November 8, 2012. [Internet].

6. Lee S-J, Jung T-Y, Lee T-R, Han $\mathrm{J}-\mathrm{H}$. Accepting telemedicine in a circulatory medicine ward in major hospitals in South Korea: Patients' and health professionals' perception of real-time electrocardiogram monitoring. $B M C$ Health Serv Res. 2018 Apr 20;18(1):
293. [PubMed] [Crossref].

7. Kravets K, Vasylenko O, Dranyk Z, Bogomolets O. Store-and-forward teledermatology for the most common skin neoplasms in Ukraine. Acta Dermatovenerol Alp Pannonica Adriat. 2018 Jun;27(2):79-83. [PubMed]

8. Jeon EM, Seo HJ. Acceptability of service targets for ICT-based healthcare. Healthc Inform Res. 2016 Oct;22(4):333-341. [PubMed] [Crossref]

9. Muigg D, Kastner P, ModreOsprian R, Haluza D, Duftschmid G. Is Austria Ready for Telemonitoring? A Readiness Assessment Among Doctors and Patients in the Field of Diabetes. Stud Health Technol Inform. 2018; 248:322-329. [PubMed]

10. Tchero $H$, Kangambega $P$, Briatte C, Brunet-Houdard S, Retali GR, Emmanuel Rusch E. Clinical Effectiveness of Telemedicine in Diabetes Mellitus: A Meta-Analysis of 42 Randomized Controlled Trials. Telemed $J$
E Health. 2019 Jul; 25(7):569-583. [PubMed] [Crossref]

11. Atallah N, Khalifa M, El Metwally A, Househ M. The prevalence and usage of mobile health applications among mental health patients in Saudi Arabia. Comput. Methods Programs Biomed. 2018 Mar; 156:163-168. [PubMed] [Crossref].

12. Krzystanek M, Borkowski M, Krysta K. Psychiatry as a leader of contemporary telemedicine in Poland. Reumatologia. 2018; 56(2):65-66. [PubMed] [Crossref].

13. LaBelle B, Franklyn AM, Nguyen VPkh, Anderson KE, Eibl JK, Marsh DC. Characterizing the Use of Telepsychiatry for Patients with Opioid Use Disorder and Cooccurring Mental Health Disorders in Ontario, Canada. Int J Telemed Appl. 2018 Feb11;2018: 7937610. [PubMed] [Crossref]

14. Fox S, Duggan M. Tracking for Health. Pew Res Cent. 2013 Jan:1-40. [Internet]

Please cite this article as: Kilova K, Milkov D, Mateva N. Attitudes Towards the Use of Information and Communication Technologies in Healthcare. J of IMAB. 2020 Jul-Sep;26(3):3317-3322.

DOI: https://doi.org/10.5272/jimab.2020263.3317

\author{
Address for correspondence: \\ Kristina Kilova, PHD \\ Department of Medical Informatics, Biostatistics and e-Learning, Faculty of \\ Public Health, Medical University of Plovdiv, Bulgaria \\ 15A, Vasil Aprilov Blvd., 4002, Plovdiv, Bulgaria \\ E-mail: k_kilova@yahoo.com,
}

